\title{
Determinantes de la eficiencia en la captación de turismo cultural nacional y extranjero en España: Un análisis regional
}

\author{
MAfalda Gómez Vega ${ }^{\text {a }}$, LUis CÉSAR HerRero Prieto ${ }^{\text {a }}$ \\ a Universidad de Valladolid, Facultad de Comercio, Plaza del Campus, 1, 47011 Valladolid, España. \\ E-mail: mafalda.gomez@uva.es, herrero@emp.uva.es
}

\begin{abstract}
RESUMEN
Planteamos un estudio fundamentado en la metodología de evaluación de la eficiencia en dos etapas sobre destinos turísticos, con una aplicación a las regiones españolas y considerando el flujo específico de turistas culturales como principal output a maximizar. El objetivo es contrastar si la concentración de recursos culturales favorece la eficiencia de los destinos turísticos en la atracción de turistas y examinar las posibilidades de intervención y ajuste en este campo. Consideramos dos modelos de estudio según el origen del flujo de turistas, nacionales e internacionales. La metodología consta de un procedimiento en dos etapas, en primer lugar se estiman los ratios de eficiencia por medio de métodos no paramétricos, y posteriormente contrastamos cómo estos ratios pueden verse condicionados por una serie de variables externas de tipo dotacional y cultural, a través de un análisis de regresión.
\end{abstract}

Palabras clave: Turismo cultural, análisis regional, análisis envolvente de datos, evaluación de la eficiencia en dos etapas.

\section{Drivers of Efficiency in Attracting Domestic and Foreign Cultural Tourism to Spain: A Regional Analysis}

\begin{abstract}
We posit a study based on the two-step efficiency evaluation method for tourist destinations, applied to Spanish regions and taking into account the specific flow of cultural tourists as the principal output to be maximised. The aim is to ascertain whether the concentration of cultural resources favours the efficiency of tourist destinations when attracting tourists and to explore the possibilities of intervention and adjustment in this field. We put forward two study models depending on whether the origin of the tourist flow is domestic or international. The method consists of a two-step procedure. First, the efficiency ratios are estimated by means of non-parametric methods. We then examine how these ratios might be shaped by a series of endowment and cultural type external variables, applying regression analysis.
\end{abstract}

Keywords: Cultural Tourism, Regional Analysis, Data Envelopment Analysis, Efficiency Evaluation, Two Stage Procedure.

Clasificación JEL: Z11,018, L83, R38, D24

Artículo recibido en enero de 2017 y aceptado en agosto de 2017

Artículo disponible en versión electrónica en la página www.revista-eea.net, ref. ə-35311 


\section{INTRODUCCIÓN}

Medir la competitividad de los destinos turísticos en cuanto a su capacidad para atraer flujos de turistas, supone una línea de investigación reciente en la economía del turismo que sin embargo ha demostrado, a tenor del número creciente de publicaciones en la materia, un interés y resultados notables (Cracolici et al., 2008; Botti et al., 2009, Suzuky et al., 2011). El desarrollo de este campo de trabajo se debe, en buena medida, a que proporciona un mayor conocimiento de la distribución territorial de la industria y la demanda de turismo, así como en el interés de comprobar el resultado de determinadas estrategias de emprendimiento productivo o dotación de recursos turísticos sobre la atracción de mayores flujos de turismo. En nuestro estudio tratamos de acotar el enfoque, ya que no analizamos el sector turístico en su totalidad, sino que vamos a abordar el particular del turismo cultural dentro de las regiones españolas. Existe un importante número de investigaciones que ubican la cultura y el patrimonio dentro de los principales atractivos que determinan las entradas de flujos turísticos (Cuccia y Cellini, 2006; Cuccia et al., 2013). Siguiendo esta línea, tratamos de constatar hasta qué punto la concentración de atractivos culturales puede determinar el grado de eficiencia con el que las regiones son capaces de atraer turistas. El resultado de este trabajo ofrece una herramienta de gestión turística, que contribuye al planteamiento de prácticas más eficientes en relación al uso y manejo de los recursos culturales, entendidos como factores de la atracción turística y en consecuencia del potencial desarrollo económico a escala regional.

Debemos puntualizar que, cuando hablamos de turistas culturales, aludimos a aquellos viajeros que realizan al menos una pernoctación (no se incluye, por tanto, a excursionistas) y que declaran como razón principal de su visita una motivación de carácter cultural. En base a la información disponible el flujo de turistas culturales nacionales, declaran estrictamente una motivación cultural de su viaje, y que representan el $14 \%$ del total de turistas nacionales; en tanto que para los turistas de origen internacional se dispone información de aquellos que declaran realizar explícitamente consumos culturales durante su viaje, y que alcanzan el $16.9 \%$ del total de turistas extranjeros ${ }^{1}$.

Basado en estas premisas, planteamos la realización de un estudio de evaluación de la eficiencia de destinos turísticos en España, tomando las diecisiete Comunidades Autónomas como unidades de análisis, abarcando el lapso temporal 2004-2012, con un enfoque basado en la metodología de análisis de eficiencia en dos etapas (Simar y Wilson, 2000; Daraio y Simar, 2005) En primer lugar se realizará un cómputo de ratios eficiencia por medio del método no

${ }^{1}$ Datos extraídos del Anuario de Estadísticas Culturales (Ministerio de Educación, Cultura y Deporte, 2013). 
paramétrico Análisis Envolvente de Datos (DEA), y posteriormente un análisis de regresión, a fin de observar la posible influencia de variables exógenas.

Partimos de una función de producción hipotética, que pone en relación el output medido en términos de flujo turístico de carácter cultural con una serie de inputs. En nuestro caso hemos considerado como factores productivos, recursos culturales que tratan de medir el capital cultural de cada una de las regiones: número de festivales, instituciones museísticas y declaraciones de Bienes de Interés Cultural (BIC). Con ello tratamos de abarcar el amplio abanico de prototipos culturales, integrando variables de patrimonio histórico, artes escénicas y musicales y las principales entidades de gestión del patrimonio cultural, los museos. Además incluimos una variable de factor trabajo como es el empleo cultural. En la denominada segunda etapa, observaremos que la eficiencia puede verse condicionada por una serie de variables externas o de entorno, por medio de la aplicación de un análisis de regresión. Consideraremos para ello la influencia de aquellos factores alusivos a la accesibilidad de las regiones, opciones de ocio alternativas, existencia de recursos de tipo natural, equipamientos hoteleros y alcance del sector en términos de empresas culturales.

Basado en esta metodología en dos etapas, proponemos la estimación de dos modelos. La diferencia entre ambos vendrá dada por el output tenido en consideración: por un lado analizaremos la eficiencia en la captación de flujos turísticos culturales provenientes del propio país, denominados en adelante como turistas culturales nacionales. Por otro lado se realizará una aplicación similar para los turistas culturales cuya procedencia sea internacional, a los que aludiremos como turistas culturales internacionales. Esta estimación se realizará con el objetivo de observar los diferentes patrones de comportamiento de cada uno de los flujos turísticos. La aplicación resulta especialmente oportuna, ya que se debe tener en cuenta que, pese a ser dos flujos bien diferenciados, los recursos culturales desplegados por las regiones, y que se consideran condicionante principal en su atracción, son los mismos para ambos casos, si bien dependiendo de las propias motivaciones, la eficiencia puede verse influenciada por unas u otras variables internas o externas, como trataremos de comprobar en la aplicación metodológica.

El artículo se organiza del siguiente modo. Tras esta introducción, se realiza una breve aproximación bibliográfica al campo de estudio, con una revisión de las publicaciones que acometen aplicaciones similares. Posteriormente se plantea la propuesta metodológica y un breve análisis del campo de estudio. El cuarto apartado trata el estudio empírico basado en la aplicación del análisis de eficiencia en dos etapas. Y para terminar, plantearemos una serie de conclusiones y puntos de discusión. 


\section{MARCO TEÓRICO COMPARADO}

El presente estudio se enmarca dentro de las investigaciones que tienen por objeto el análisis del turismo cultural, analizado bajo el punto de vista de la eficiencia. Contamos con un amplio y contrastado número de ejemplos en torno a la evaluación de servicios públicos dentro de sectores como el educativo, el sanitario o el de la investigación (Urueña y Martín, 2012; Quindós et al., 2005; Castrodeza y Peña, 2002). Por su parte el sector cultural ha ido recibiendo, en este sentido, una mayor atención en los últimos años, si bien aún se encuentra lejos de contar una frecuencia de estudios similar (Fernández Blanco et al., 2013). Contamos con diferentes ejemplos de aplicaciones de la metodología de frontera de producción estocástica por medio de modelos paramétricos, dentro del contexto de la evaluación de instituciones culturales como los museos (Bishop y Brand, 2003) y los teatros, (Zieba, 2013; Last y Wetzel, 2010). Sin embargo, son más frecuentes las aplicaciones con empleo de técnicas no paramétricas, fundamentalmente DEA, siendo los museos el principal objeto de estudio, con ejemplos de estudios en Bélgica (Mairesse y Eeckaut, 2002), Italia (Pignataro, 2002; Basso y Funari, 2004) y España (del Barrio et al., 2009; del Barrio y Herrero, 2014). En cuanto a aplicaciones con modelo en dos etapas, son interesantes los estudios centrados en la evaluación de la eficiencia de las bibliotecas (Vitaliano, 1998; de Witte y Geys, 2011) y agencias de patrimonio para el caso italiano (Finochiaro et al., 2011)

Si acotamos la búsqueda de estudios dentro del particular del turismo, nos encontramos con que en los años más recientes ha sido un terreno de estudio bastante fecundo, que ha dado lugar, incluso, a estudios de síntesis científica sobre esta línea de investigación (Sainaghi, et al., 2017). La eficiencia de las agencias de viajes es una de las unidades de análisis más tenida en cuenta, con ejemplos en el marco español (Fuentes, 2011), Turquía (Köksal y Aksu, 2007) y Portugal (Barros y Matias, 2006). Si bien, es el sector hotelero el que aglutina mayor número de estudios, enfocados desde diferentes perspectivas y con enfoques metodológicos variados, como la metodología de frontera estocástica dentro de la oferta hotelera portuguesa (Barros, 2004 y 2006) y sobre este mismo sector en Italia (Brida et al., 2015), o Eslovenia (Assaf y Cvelbar, 2010). Sin embargo, de nuevo constatamos que la mayoría de los ejemplos son aquellos que usan técnicas no paramétricas para evaluar la eficiencia, principalmente DEA, como es el caso de los hoteles en Reino Unido (Sigala, 2004) o las pousadas en Portugal (Barros, 2005) o en el sector hotelero de las Islas Baleares en España (Riera et al., 2011). Además existen diferentes estudios que avanzan en los modelos de eficiencia condicionada y complementan la metodología DEA, por medio del modelo de regresión Tobit, aplicados al sector hotelero en países como Taiwan o Australia (Shang et al., 2010; Wang, 2006; Assaf y Agbola, 2011).

Por último aludimos a aquellos ejemplos que se ciñen de forma específica al 
campo de nuestra aplicación, es decir, la eficiencia de regiones como destinos turísticos. En la mayoría de estos casos se encuentran aplicaciones de metodología en dos etapas, siguiendo la propuesta de Simar y Wilson (2007). Generalmente desarrollan un análisis DEA posteriormente los resultados de la eficiencia son considerados en una regresión a modo de variable dependiente, junto a toda una serie de variables externas o de entorno, para contrastar su efecto condicionante (Dios et al., 2006). En la fase del análisis de eficiencia es bastante habitual considerar una función de producción sencilla que relaciona las pernoctaciones en función la capacidad hotelera y la llegada de flujos turísticos. En la segunda etapa, se tienen en consideración un amplio abanico de variables, como los recursos culturales, seguridad, accesibilidad, etc. (vid. Barros et al., 2011, Botti et al., 2009, Benito et al., 2014). Además existen otra serie de trabajos que proponen, por el contrario, una función de producción más compleja, introduciendo algunas variables, anteriormente consideradas como factores externos, dentro de la propia función de producción (Cracolici et al., 2008 y Suzuky et al., 2011). Este es el ámbito en el que se engloba la presente propuesta. En nuestro caso algunos factores son introducidos como inputs explicativos de la eficiencia técnica, especialmente variables referentes a recursos culturales, que se consideran los principales determinantes de la atracción de este tipo de turismo.

\section{METODOLOGÍA Y CASO DE ESTUDIO}

Contamos con un importante número de publicaciones que abordan la eficiencia de los destinos turísticos de forma global, es decir, sin realizar acotaciones alrededor de los motivos de los turistas para realizar su viaje. En este trabajo se considera que las diferentes motivaciones del turismo deben ser tenidas en cuenta, ya que al hacerlo pueden variar de forma sustancial los ratios de eficiencia de las regiones observadas, al igual que las conclusiones que de ello pueden extraerse en términos de posibilidades de actuación e intervención sobre los recursos y atractivos turísticos. En consecuencia, este nuevo punto de vista puede arrojar resultados sustancialmente distintos a lo que se ha venido realizando hasta el momento (Benito et al., 2014) No obstante, plantear esta precisión analítica implica desarrollar una función de producción específica y compleja, frente a las que con anterioridad ponían en relación variables puramente de gestión de la industria turística, es decir, pernoctaciones en función de la capacidad hotelera y el flujo de turistas.

En nuestro planteamiento metodológico se introduce, por tanto, una acotación especialmente significativa (Herrero y Gómez, 2017), que es considerar como output de la función de producción el flujo de turistas culturales, mientras que del lado de los inputs se toman en cuenta variables relativas a los recursos culturales disponibles en cada región, principales determinantes de su visita, tratando de optar por las que mejor sinteticen los atractivos culturales de las regiones. En 
este sentido hemos seleccionado los siguientes: el número de festivales culturales en el ámbito escénico, musical o cinematográfico, expresión de la actividad artística corriente de cada región; el número de museos, institución por excelencia de gestión del patrimonio cultural; y por último, los bienes declarados Bien de Interés Cultural (BIC), ejemplo de dotaciones de patrimonio histórico con especial notoriedad. Además se incluye como variable representativa del factor trabajo dentro de la función de producción, el número de empleados del sector cultural regional.

En cuanto a la fuente de información, tanto de variables de oferta como de demanda y de actividad, proviene del INE a través de la explotación CulturaBase del Ministerio de Educación, Cultura y Deporte, y las estadísticas propias del Instituto de Estudios Turísticos (FAMILITUR, movimientos turísticos de los españoles, y FRONTUR, movimientos turísticos en fronteras). Cabe destacar que, debido a la diferente naturaleza de los datos, para el caso de los turistas culturales nacionales se consideran aquellos que expresan una motivación específicamente cultural en su desplazamiento, mientras que en el caso de los turistas culturales internacionales se incluyen a todos aquellos que realizan consumos culturales, cualquiera que sea la finalidad de su viaje. Este es el principal motivo por el que ambos datos no pueden unificarse y compararse de forma directa, aunque estén estrictamente ligados pues se vinculan en la afinidad cultural, entendida como motivo exclusivo del viaje en el caso de los turistas nacionales, o como consumo frecuente durante la visita en el caso de los turistas internacionales. Por ello, ambos flujos se analizan de manera diferenciada en nuestro trabajo y se trata de dos estudios paralelos, pero vinculados por afinidad. Se considera por lo tanto sendas matrices muestrales de 5 variables que conforman la función de producción (4 inputs y 1 output) para 17 Comunidades Autónomas durante 5 años del intervalo temporal 2004-2012 y para cada segmento de turistas, nacional o internacional ${ }^{2}$. Trabajamos con datos panel, de modo que cada una de las Comunidades Autónomas en cada año, es considerada como una unidad distinta. De este modo estamos generando dos bases de datos de 425 observaciones para cada tipo de turismo.

La metodología que sustenta nuestro análisis se basa en los modelos de eficiencia condicionada en dos etapas. En una primera fase por medio del DEA, aplicado sobre la función de producción anteriormente definida, para medir el ratio de eficiencia técnica de los destinos de turismo cultural. La metodología DEA es una técnica de programación lineal, que evalúa el nivel de eficiencia de un grupo de unidades de toma de decisión, en nuestro caso regiones, calculando

\footnotetext{
${ }^{2}$ En cuanto al periodo analizado cabe matizar que incluimos únicamente los años pares, ya que solo para ellos contamos con la serie completa de datos, para todas las variables y todas las regiones. Esta es la razón por la que se ha tomado el periodo completo de análisis, y no se ha considerado una posible división en periodos ajustados al ciclo económico.
} 
una envolvente con las unidades que presentan las mejores prácticas o resultados, así como sus combinaciones lineales, dejando por debajo las unidades ineficientes. Largo se ha estudiado sobre las ventajas de aplicar esta metodología, especialmente útil en la evaluación de la provisión de bienes y recursos culturales (Fernández Blanco et al., 2013) debido a que no requiere indicar la forma específica de la función de producción hipotética, puesto que emplea únicamente la información empírica que conoce, resolviendo un simple problema de optimización. Sin embargo, es importante saber que también presenta varias desventajas, como que debido a su carácter determinístico, considera no eficientes todas aquellas observaciones que se alejen de la frontera eficiente, sin poder atribuirlo a algún otro factor aleatorio que condicione el proceso de producción. Además se revela especialmente importante la selección de unidades analizadas, siendo necesario que éstas muestren una cierta homogeneidad, para evitar que la posible disparidad afecte a las estimaciones (Martínez, 2003). Estas posibles desventajas tratan de solventarse precisamente con un análisis de segunda etapa, donde se estudia el efecto de distintas variables externas sobre la eficiencia de las unidades consideradas a través de un análisis de regresión donde los ratios de eficiencia son la variable dependiente y los factores de entorno sirven como variables explicativas (Banker y Natarajan, 2008)

Otra de las utilidades de la metodología DEA es la detallada información que ofrece de las unidades ineficientes, calculando su ineficiencia relativa como la distancia que las separa hasta la frontera de eficiencia (Álvarez, 2001). A lo que se suma la posibilidad de caracterizar esta ineficiencia, dado que aporta información de las mejoras que estas unidades ineficientes deben acometer para alcanzar la frontera óptima. Es decir, en un modelo orientado al input, obtendremos una medida relativa de la reducción de los mismos que debería acometerse, dado un nivel de output, a fin de alcanzar el óptimo de eficiencia. De igual manera en el caso opuesto, en un modelo con orientación al output, podremos obtener una medida de la potencial mejora que las unidades ineficientes pueden alcanzar en esta variable, según su nivel de input. Será esta segunda orientación la aplicada en el presente trabajo descrito matemáticamente del siguiente modo.

Consideramos $n$ DMU a evaluar. El DEA orientado al output calcula un resultado $\theta_{i}$ para cada una de las DMU, dando solución al programa lineal $i=1, \ldots ., n$, bajo el supuesto de rendimientos a escala constantes (CCR):

$$
\begin{aligned}
& \text { Max } \lambda \theta_{i} \omega_{i} \\
& \text { Sujeto a } x_{i}-X \lambda \geq 0 \\
& Y \lambda-\omega_{i} y_{i} \geq 0 \\
& \lambda \geq 0
\end{aligned}
$$

donde $x_{i}$ e $y_{i}$ son respectivamente el input y el output de $i$ DMU; $\mathrm{X}$ es la matriz 
del input, mientras que $\mathrm{Y}$ es la matriz del output, y $\lambda$ es un vector de $n \times 1$ variables. El modelo [1] puede variarse a fin de considerar rendimientos a escala variables (BCC), si se incluye la restricción de convexidad: $e \lambda=1$. Donde $e$ es un vector con todos sus elementos iguales a 1, lo que incluye la llamada Eficiencia Técnica Pura ${ }^{3}$. Además siguiendo a Simar y Wilson (2000) y con la finalidad de ofrecer una prueba de la robustez de nuestras estimaciones, completamos el modelo por medio del procedimiento Bootstrap con 2.000 iteraciones, para eliminar posibles sesgos, y obtener la varianza y los intervalos de confianza de los índices calculados por el DEA. En cuanto a la segunda etapa ${ }^{4}$, una vez se han obtenido los ratios de eficiencia, estos son incluidos como variable dependiente en un análisis de regresión. Las variables explicativas o independientes, son aquellas que representan inputs no controlables, y que inicialmente son excluidos de la primera etapa. Si bien es posible que contribuyan a explicar posibles ineficiencias, no pueden ser incluidas dentro de la función de producción ya que son variables de entorno, no discrecionales, que no forman parte del proceso productivo en sí mismo. Sin embargo no contradice que su incidencia pueda condicionar los resultados y por consiguiente, las conclusiones extraídas. Habitualmente se consideran factores climáticos, geográficos, etc., pero en nuestro estudio vamos a introducir variables de dotaciones, servicios, y de ocio alternativo, que junto a los factores de la primera etapa se detallan en la Tabla 1.

En primer lugar, hemos considerado el número de declaraciones de Patrimonio de la Humanidad por la UNESCO, tratando de captar la posible tendencia de los turistas a viajar a aquellas regiones con elementos patrimoniales de mayor relevancia y popularidad. A la hora de conformar esta variable no solo se tiene en cuenta cada declaración, sino que han sido además ponderadas por el número de años desde su inclusión en la lista de bienes declarados. De esta manera vamos a incorporar a la estimación tanto el número de declaraciones como la antigüedad de las mismas, lo cual puede ir correlacionado con una mayor notoriedad. Por otro lado se ha considerado oportuno incluir toda una serie de variables que aluden al grado de accesibilidad turística de la que goza cada región, tanto en términos de capacidad hotelera (camas en establecimientos hoteleros y en casas rurales) con una diferenciación ( 3,4 y 5), como en términos de kilometraje de autopistas en relación al tamaño de la región, ejemplo de acceso rápido y cómodo

\footnotetext{
${ }^{3}$ El modelo CCR mide la eficiencia global de cada unidad (Eficiencia Técnica Pura y la Eficiencia de Escala), mientras que el modelo BBC estima la medida de la Eficiencia Técnica Pura. Añadir que la estimación de la Eficiencia de Escala puede obtenerse del cociente del resultado del modelo CCR y el del BBC. Para más información al respecto puede consultarse Martínez (2003).

${ }^{4}$ Los principales artículos que han sido referentes en el procedimiento en dos etapas son los realizados por Simar y Wilson (2000 y 2007) y para el caso específico de los destinos culturales Cuccia et al. (2013).
} 
a los diferentes ofertas culturales. En tercer lugar abordamos la posible naturaleza omnívora del turista cultural, es decir el consumo de otro tipo de actividades en paralelo a lo propiamente cultural. Esto trata de expresarse por medio de las variables superficie de parques naturales y el kilometraje de costa, entendiendo por lo tanto, que ambos elementos (parques naturales y playas) pueden actuar como factores sustitutivos frente al turismo cultural, o también en algunos casos como bienes complementarios, es decir, turismo omnívoro (Barbieri y Mahoney, 2010). En cuarto lugar se incluyen variables alusivas al alcance del sector cultural a nivel regional. La primera de ellas será el gasto público en cultura que realiza el gobierno autonómico, además del número de empresas culturales asentadas en cada región, representativo del entramado productivo cultural en esta área. Estas variables pueden tener una tendencia expresiva del ciclo económico durante el periodo analizado, sobretodo la primera, gasto público cultural, con una merma significativa en la crisis económica. Las empresas culturales han tenido un comportamiento más sostenido. Por último, incluimos la variable seguridad, que engloba la actividad delictiva en cada región (indicador fruto de la agregación de la cifra de crímenes y de la de robos), posible condicionante para la llegada de turistas.

Tabla 1

Variables y estadísticos descriptivos

\begin{tabular}{|c|c|c|c|c|c|c|}
\hline & Variable & Descripción & Media & Desv. Est. & Mínimo & Máximo \\
\hline \multirow{6}{*}{ 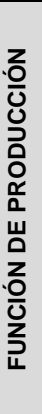 } & Festivales & $\begin{array}{l}\mathrm{N}^{\circ} \text { de festivales programados en cada } \\
\text { CC.AA. }\end{array}$ & 124.1 & 109.5 & 21.0 & 409.0 \\
\hline & Museos & $\mathrm{N}^{\circ}$ de museos en cada CC.AA. & 87.4 & 59.3 & 8.0 & 206.0 \\
\hline & $\begin{array}{l}\text { Patrimonio } \\
\text { Histórico }\end{array}$ & $\begin{array}{l}\mathrm{N}^{\circ} \text { de elementos de patrimonio histórico } \\
\text { protegidos (BICs) en cada CC.AA. }\end{array}$ & 798.4 & 835.3 & 103.0 & 2890.0 \\
\hline & $\begin{array}{l}\text { Empleo } \\
\text { Cultural }\end{array}$ & $\begin{array}{c}N^{\circ} \text { de empleados en el sector cultural (en } \\
\text { miles) }\end{array}$ & 30.0 & 36.9 & 3.8 & 139.1 \\
\hline & $\begin{array}{l}\text { Turismo } \\
\text { Cultural } \\
\text { Nacional }\end{array}$ & \begin{tabular}{|c|}
$N^{0}$ de turistas nacionales que realizan \\
viajes dentro de España con motivación \\
cultural (en miles)
\end{tabular} & 500477,6 & 381858,2 & 61500 & 1795000 \\
\hline & $\begin{array}{c}\text { Turismo } \\
\text { Cultural } \\
\text { Internacional }\end{array}$ & $\begin{array}{l}N^{\circ} \text { de turistas internacionales que } \\
\text { realizan viajes dentro de España con } \\
\text { motivación cultural (en miles) }\end{array}$ & 1748726,47 & 2360224,53 & 45200 & 9776800 \\
\hline \multirow{6}{*}{ 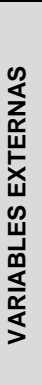 } & Autopistas & \begin{tabular}{|c|} 
Kilómetros de autopista en cada CC.AA. \\
por $100 \mathrm{~km} 2$
\end{tabular} & 4.1 & 2.5 & 1.0 & 12.3 \\
\hline & Seguridad & $\begin{array}{c}N^{\circ} \text { de robos y delitos en cada CC.AA. por } \\
1000 \mathrm{~km} 2\end{array}$ & 16.6 & 17.2 & 1.2 & 83.5 \\
\hline & $\begin{array}{l}\text { Empresas } \\
\text { Culturales }\end{array}$ & $\begin{array}{c}\mathrm{N}^{\circ} \text { de empresas dedicadas a la cultura } \\
\text { en cada CC.AA. por } 100 \mathrm{~km} 2\end{array}$ & 32.6 & 55.7 & 2.1 & 288.9 \\
\hline & Gasto cultural & $\begin{array}{l}\text { Gasto cultural del gobierno de cada } \\
\text { CC.AA. por } 1000 \text { habitantes }\end{array}$ & 42.6 & 21.4 & 9.0 & 126.7 \\
\hline & Costa & \begin{tabular}{|c|} 
Kilómetros de playa en cada CC.AA. por \\
$100 \mathrm{~km} 2$
\end{tabular} & 4.4 & 7.8 & 0 & 28.6 \\
\hline & $\begin{array}{l}\text { Plazas } 345 \\
\text { Estrellas }\end{array}$ & $\begin{array}{l}\mathrm{N}^{\circ} \text { de camas en hoteles de } 3,4 \text { o } 5 \\
\text { estrellas en cada CC.AA. por km2 }\end{array}$ & 3.3 & 6.1 & 0.1 & 29.7 \\
\hline
\end{tabular}


Tabla 1 (Continuación)

Variables y estadísticos descriptivos

\begin{tabular}{|c|c|c|c|c|c|c|}
\hline & Variable & Descripción & Media & Desv. Est. & Mínimo & Máximo \\
\hline \multirow{5}{*}{ 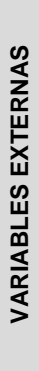 } & Plazas Totales & $\begin{array}{l}\mathrm{N}^{\circ} \text { de camas disponibles en } \\
\text { establecimientos hoteleros en cada } \\
\text { CC.AA. por km2 }\end{array}$ & 3.6 & 6.3 & 0.2 & 30.6 \\
\hline & Plazas rural & $\begin{array}{l}\mathrm{N}^{\circ} \text { de camas en hoteles rurales en cada } \\
\text { CC.AA. por km2 }\end{array}$ & 33.9 & 26.0 & 1.4 & 112.3 \\
\hline & $\begin{array}{l}\text { Parques } \\
\text { naturales }\end{array}$ & $\begin{array}{c}\text { Superficie de los parques naturales en } \\
\text { cada CC.AA. por } 1000 \mathrm{~km} 2\end{array}$ & 9.0 & 12.6 & 0 & 44.0 \\
\hline & Tendencia & $\begin{array}{c}\text { Factor temporal que indica el caso de los } \\
\text { años objeto de la muestra }\end{array}$ & 8 & 2.84 & 4 & 12 \\
\hline & $\begin{array}{l}\text { Declaraciones } \\
\text { UNESCO }\end{array}$ & $\begin{array}{l}\mathrm{N}^{\circ} \text { de declaraciones UNESCO, en cada } \\
\text { CC.AA. ponderado por años de } \\
\text { inscripción }\end{array}$ & 44.7 & 30.4 & 6.0 & 134.0 \\
\hline
\end{tabular}

Fuente: Elaboración propia.

Los resultados de la eficiencia estimados en la primera etapa, se introducen en un análisis de regresión como variable dependiente, y los factores externos como variables independientes, conformando una regresión de datos trasversales especificada de la siguiente manera:

$$
\theta_{i}=f\left(z_{i}\right)+\varepsilon_{i}
$$

Donde $\theta_{i}$ representa el índice de eficiencia extraído del DEA, $z_{i}$ está conformado por las variables ambientales, y $\varepsilon_{i}$ es un vector que recoge el posible error. Para evitar posibles sesgos se aplica la técnica de bootstrapping. Una vez aplicada esta corrección se estima una regresión truncada (Simar y Wilson, 2007; Benito et al., 2014) que nos permitirá observar qué variables determinan la eficiencia de las regiones, en qué medida lo hacen según la interpretación de su coeficiente, y si su efecto condicionante actúa de forma positiva o negativa, dependiendo del signo de ese coeficiente. El análisis en dos etapas permite caracterizar las posibles ineficiencias que puedan ser únicamente fruto del carácter determinístico del DEA, señalando hacia los factores externos que la condicionan.

Como paso previo a la implementación de la metodología, resulta interesante realizar una breve alusión de los datos que vamos a manejar, a modo de contextualización del sector en el que queda inserto el trabajo, la distribución regional del turismo cultural y los recursos culturales. En este sentido, y a la vista de la Figura 1, podemos señalar que la entrada de turistas culturales internacionales, en sintonía con la cifra de turistas generales, se ha visto sensiblemente afectada por la crisis económica de 2008. Sin embargo, una mirada más detallada nos indica que la cifra de turistas nacionales, con motivación estrictamente cultural, ha permanecido relativamente estable en los últimos años, con un efecto retardado de la crisis económica, puesto que se 
denota un retroceso del flujo a partir de 2011. Por su parte, los turistas internacionales con consumos culturales, sufren un retroceso notable entre 2008 y 2011, para repuntar posteriormente, a ritmo anual importante. Los destinos preferidos por el flujo de turistas son, para el caso del turismo nacional, sobre todo Andalucía, seguida de Cataluña y Castilla y León. En el lado contrario, las regiones menos frecuentadas por los turistas nacionales son Murcia y La Rioja. Entre los turistas de origen internacional, destacan con mayor número de entradas, las regiones de Cataluña, Andalucía y Canarias, mientras que La Rioja y Castilla la Mancha presentan las cifras más bajas.

Figura 1

Evolución de los turistas culturales en España según origen

(En miles)

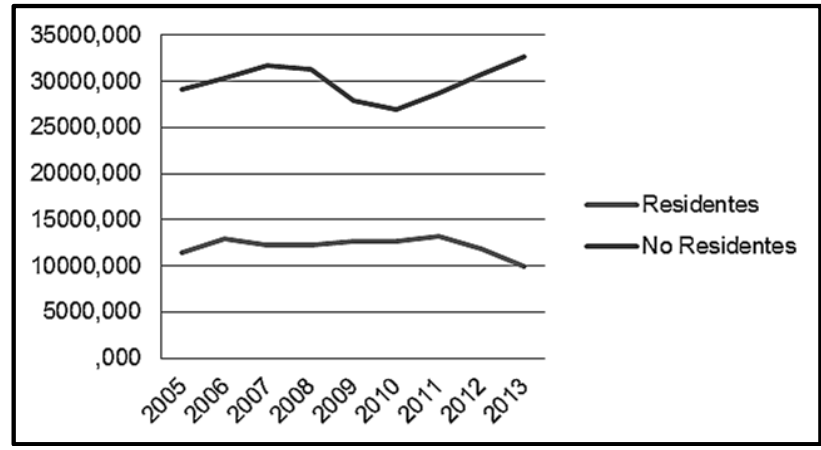

Fuente: Elaboración propia a través de CULTURAbase.

Figura 2

Evolución de los recursos culturales en España

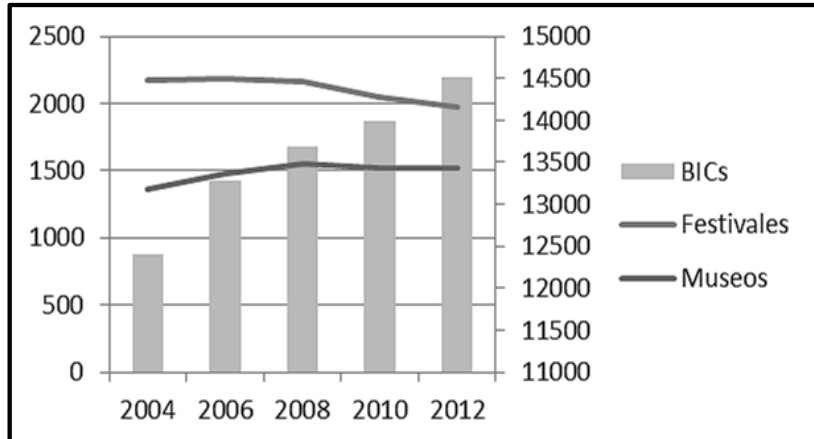

Fuente: Elaboración propia a través de CULTURAbase.

En cuanto a la evolución de los factores productivos considerados en la función de producción, (Figura 2) solo apuntar que a medida que pasan los años, las cifras tienen a estancarse, sino a retroceder a partir de la crisis económica de 
2008 en el número de festivales y museos. No ocurre así en el número de BICs (Bienes de Interés Cultural), que continúa creciendo progresivamente debido, principalmente, a que la declaración de expedientes de BIC no requiere de recursos financieros sino de la simple voluntad institucional de reconocimiento. Resulta interesante, por lo tanto, examinar la evolución de la eficiencia regional en la captación de turismo cultural en estos últimos años de recesión ligera de la demanda y contención de algunos de los elementos de la oferta cultural.

\section{APLICACIÓN EMPÍRICA}

En primer lugar se acomete la aplicación del DEA, para obtener los resultados de la eficiencia de los destinos en cuanto a la atracción de turistas culturales. Partiendo de la función de producción anteriormente diseñada para cada modelo, formada por inputs comunes de capital cultural (Festivales, Museos, BICs) y de empleo cultural, la diferencia viene dada por el lado de los outputs: en el primer modelo (Modelo A) consideraremos el número de turistas culturales nacionales y en el segundo (Modelo B) los turistas culturales internacionales.

De forma previa a la aplicación, debemos caracterizar el modelo dentro de las diferentes opciones que la metodología permite, adecuándolo a la naturaleza de la muestra, y al tipo de conclusiones que pretendemos extraer. En primer lugar, condicionado por el tamaño de la muestra, realizamos las estimaciones en base a un panel de datos, es decir, todas las regiones son tomadas como unidades distintas en cada año considerado. En cuanto a las especificaciones del propio DEA, y dado el carácter de los recursos y variables con las que vamos a trabajar, hemos optado por el modelo orientado hacia la maximización del output, es decir, que se considerarán eficientes aquellas regiones que, dado un nivel de input (empleo y recursos culturales), alcanzan el máximo output (turistas culturales), siendo las que conformarán la frontera de casos eficientes. Se trata del modelo más habitual dentro de los análisis de eficiencia del sector turístico (Cracolici et al., 2008; Barros et al., 2011) y especialmente en las aplicaciones de eficiencia en dos etapas. En cuanto a la hipótesis técnica, nos inclinamos por el modelo de rendimientos constantes a escala (CCR) ya que nos aporta información de la eficiencia de forma global, suma de la eficiencia técnica y de escala y también es ampliamente utilizado en estos estudios (Cuccia et al., 2013) 5 .

Aplicado el DEA sobre los dos modelos A y B, para las 17 Comunidades Autónomas, bajo la hipótesis tecnológica de rendimientos a escala constantes (CCR) y con una orientación a la maximización del output, obtenemos los siguientes resultados. En la Tabla 2 se presentan los porcentajes de eficiencia alcanzados por cada unidad. Apuntar que, a pesar de que la estimación se

\footnotetext{
${ }^{5}$ No obstante, se ha estimado el modelo bajo el supuesto alternativo BCC, con rendimientos de escala variables, resultando mucho menos restrictivo ya que las unidades consideradas eficientes en el modelo CCR también lo serán en el BCC, pero no al revés.
} 
realiza en base a un panel de datos, con el objetivo de facilitar su comprensión, los resultados se presentan en una estructura longitudinal por años, calculando finalmente la media del periodo.

Tabla 2

Ratio de eficiencia por destinos turísticos culturales 2004-2012

\begin{tabular}{|c|c|c|c|c|c|c|c|c|c|c|c|c|}
\hline & \multicolumn{6}{|c|}{ Modelo A } & \multicolumn{6}{|c|}{ Modelo B } \\
\hline & 04 & 06 & 08 & 10 & 12 & $\begin{array}{c}\text { CCR } \\
\text { Medio }\end{array}$ & 04 & 06 & 08 & 10 & 12 & $\begin{array}{c}\text { CCR } \\
\text { Medio }\end{array}$ \\
\hline Andalucía & 45,1 & 47,5 & 41,6 & 42,5 & 51,2 & 45,6 & 47,7 & 43,8 & 40,4 & 33,2 & 37,4 & 40,5 \\
\hline Aragón & 60,7 & 71,4 & 62,2 & 64,9 & 67,2 & 65,3 & 9,5 & 6,7 & 7,4 & 5,1 & 5,6 & 6,9 \\
\hline Asturias & 65,1 & 100 & 76,1 & 69,6 & 87,3 & 79,6 & 8,4 & 8,1 & 5,9 & 6,1 & 7,3 & 7,2 \\
\hline Islas Baleares & 28,4 & 35,6 & 58,2 & 42,2 & 29,7 & 38,8 & 100 & 97,3 & 97,4 & 89,3 & 100 & 96,8 \\
\hline Islas Canarias & 57,2 & 45,4 & 74,2 & 69,9 & 28,6 & 55,1 & 100 & 100 & 100 & 80,9 & 100 & 96,2 \\
\hline Cantabria & 100 & 71,4 & 89,3 & 92,0 & 62,4 & 83,0 & 30,1 & 25,0 & 27,3 & 23,7 & 20,5 & 25,3 \\
\hline Castilla y León & 64,0 & 58,8 & 40,4 & 46,8 & 53,9 & 52,8 & 16,0 & 13,7 & 10,2 & 6,6 & 9,4 & 11,2 \\
\hline Castilla la Mancha & 80,1 & 46,7 & 43,6 & 38,6 & 51,7 & 52,2 & 4,8 & 3,8 & 3,1 & 2,2 & 2,9 & 3,3 \\
\hline Cataluña & 27,8 & 31,2 & 27,5 & 23,6 & 24,1 & 26,9 & 96,2 & 94,3 & 98,1 & 81,7 & 100 & 94,1 \\
\hline C. Valenciana & 42,8 & 35,9 & 30,7 & 29,8 & 20,5 & 31,9 & 26,5 & 25,9 & 26,5 & 22,8 & 22,9 & 24,9 \\
\hline Extremadura & 100 & 81,4 & 66,8 & 64,3 & 72,9 & 77,1 & 15,3 & 12,1 & 9,8 & 7,1 & 7,9 & 10,5 \\
\hline Galicia & 50,4 & 65,9 & 52,5 & 50,3 & 43,1 & 52,5 & 18,4 & 17,4 & 12,4 & 7,6 & 8,5 & 12,8 \\
\hline Madrid & 84,1 & 100 & 77,3 & 91,4 & 98,2 & 90,2 & 66,7 & 71,9 & 85,5 & 93,4 & 92,5 & 82,0 \\
\hline Murcia & 22,8 & 28,2 & 23,8 & 12,4 & 15,7 & 20,6 & 11,9 & 11,7 & 11,3 & 11,3 & 8,3 & 10,9 \\
\hline Navarra & 56,23 & 45,7 & 26,9 & 50,7 & 41,1 & 44,14 & 8,7 & 6,2 & 7,5 & 16,3 & 13,1 & 10,4 \\
\hline País Vasco & 32,7 & 33,8 & 20,56 & 41,2 & 39,4 & 33,52 & 18,1 & 19,2 & 16,3 & 14,7 & 19,7 & 17,6 \\
\hline La Rioja & 66,1 & 42,7 & 57,23 & 55,6 & 84,7 & 61,3 & 12,1 & 9,4 & 7,7 & 5,1 & 4,9 & 7,8 \\
\hline Eficiencia media & 57,9 & 55,4 & 51,1 & 52,1 & 51,3 & 53,6 & 34,7 & 33,3 & 33,3 & 29,8 & 32,9 & 32,8 \\
\hline Regiones eficientes & 2 & 2 & 0 & 0 & 0 & 0 & 2 & 1 & 1 & 0 & 3 & 0 \\
\hline
\end{tabular}

Fuente: Elaboración propia.

En el modelo A (turismo cultural nacional), observamos que la media de eficiencia a lo largo del periodo para todas las comunidades autónomas, es del $53,6 \%$, lo que nos indica que existe un margen de mejora, en cuando a la maximización del output, de 46,4 puntos porcentuales, dado el nivel de input considerado. La eficiencia media ha decaído de manera significativa hasta un curso más o menos constante al final del periodo. En el primer año analizado, 2004, tan solo dos Comunidades Autónomas son eficientes, Cantabria y Extremadura, dos regiones no especialmente turísticas y en ambos casos periféricas. La primera de ellas de la costa norte y la segunda de interior. En el año 2006 de nuevo son dos, pero en este caso Asturias en el Cantábrico y Madrid como región interior. Madrid es la capital del país, con una acumulación muy importante de instituciones culturales de rango nacional y enorme relieve, además de poseer el aeropuerto más importante y principal entrada de turistas (especialmente significativo a nivel internacional). Ambos años son los que muestran una eficiencia media más alta, en comparación a los años siguientes, 
donde no solo la eficiencia media es inferior, sino que además ninguna región se encuentra sobre la frontera de eficiencia, denotando el posible efecto de la crisis económica. Si realizamos una observación general del periodo, podemos ver que las regiones que muestran un mayor ratio de eficiencia son las denominadas "regiones de interior", Extremadura, Madrid, Aragón y La Rioja, a las que se suman dos regiones del litoral, Asturias y Cantabria, ambas de la costa norte. Este resultado comienza a apuntar hacia las características del turismo cultural que desarrollan los turistas nacionales, entre los que los consumos combinados de cultura y costa no parecen estar relacionados. Para el caso de las dos regiones de la costa del norte con un ratio de eficiencia alto, es importante caracterizar este tipo de atractivo turístico. En líneas generales muestra un patrón de consumo turístico menos masivo, condicionado por el un clima menos amable y la propia tipología de las playas, más abruptas y de acceso complicado. Las regiones con mayor margen de mejora, es decir las menos eficientes en la atracción de turistas nacionales específicamente culturales, son las de la zona del arco Mediterráneo (Cataluña, Comunidad Valenciana, Murcia y Andalucía) en contra de lo que se podría esperar a priori, dado que alguna de estas regiones aglutinan un importante porcentaje del flujo turístico cultural nacional. No obtienen un ratio de eficiencia elevado, con respecto a su nivel de recursos culturales y empleo cultural, lo que denota una cierta sobredimensión. La Figura 3 ofrece una clara imagen de la evolución de la eficiencia de las regiones del Modelo A, entre 2004 y 2012. Por un lado observamos cómo hay una serie de regiones que mejoran su ratio de eficiencia, aun cuando ya se situaban en las mejores posiciones al inicio del periodo, Madrid, La Rioja, Asturias y Aragón. El segundo lugar encontramos un grupo de regiones, Castilla la Mancha, Cantabria y Extremadura, que acusan una importante caída al final del periodo aunque se mantienen en las cifras de eficiencia media nacional. Por su parte, las regiones más retrasadas al inicio de este, se mantienen en las últimas posiciones con ratios estables, siendo estas las regiones del Arco Mediterráneo, País Vasco y Baleares.

En el modelo B (turismo cultural internacional), encontramos una situación completamente diferente. En líneas generales las posiciones se alteran, las regiones con mayor ratio de eficiencia son Cataluña y ambas regiones insulares, Baleares y Canarias, siendo estas dos últimas las únicas que alcanzan el óptimo de eficiencia. Todas ellas con un importante turismo de sol y playa, condicionado por el buen clima y el tipo de playas, que contribuye a su notoriedad internacional y las sitúa como destino preferente del turismo internacional. El resto de Comunidades Autónomas se sitúan muy por debajo de las anteriormente mencionadas, con ratios de eficiencia inferiores al 50\%, exceptuando la Comunidad de Madrid, en torno al 66\%. Esto hace que la eficiencia media acumulada del periodo sea considerablemente inferior con respecto a la del modelo A, alcanzando tan solo un 32,8\%, con un margen de mejora por lo tanto del 67,2 puntos porcentuales. Esto evidencia la orientación general del flujo de 
turistas extranjeros, la mayor parte de estos se dirige a estas tres Comunidades Autónomas, que a su vez son un buen ejemplo del carácter omnívoro de este tipo de turismo, dado que se intuye la posible tendencia a combinar la motivación cultural con otros consumos relacionados, por ejemplo el turismo de sol y playa. Trataremos a continuación, dentro del análisis de segunda etapa, de refrendar esta afirmación incluyendo variables alusivas a ese tipo de ocio alternativo o complementario. Si nos detenemos en analizar la evolución de la eficiencia entre ambos extremos del periodo, vemos en la Figura 4 que en el año 2012 se mantiene un panorama similar, con el espectacular aumento de la eficiencia de la Comunidad de Madrid que se sitúa muy cerca de la frontera óptima. En sintonía con en el anterior modelo, se percibe el efecto de la capitalidad de esta región, principal entrada de turistas por vía aérea, presencia de las dotaciones e instituciones culturales con mayor proyección internacional (Museo del Prado, Reina Sofía, etc.) Desarrollan unas prácticas eficientes de nuevo las Comunidades de Baleares, Canarias, sumándose ahora también la Comunidad de Cataluña, región con una notoriedad internacional muy elevada, protagonizada por su capital, Barcelona. El resto de regiones no solo se mantienen retrasadas con respecto a las unidades eficientes, si no que las diferencias, de forma general, tienen a presentar un sustancial aumento, ya que su porcentaje de eficiencia tiende a verse reducido. Destacan los ratios de eficiencia inferiores al 10\% de regiones como Castilla la Mancha, La Rioja, Aragón y Asturias, fundamentalmente regiones de interior y con menor notoriedad a nivel internacional.

Figura 3

Modelo A. Turismo Cultural Nacional. Evolución de los ratios de eficiencia 2004-2012

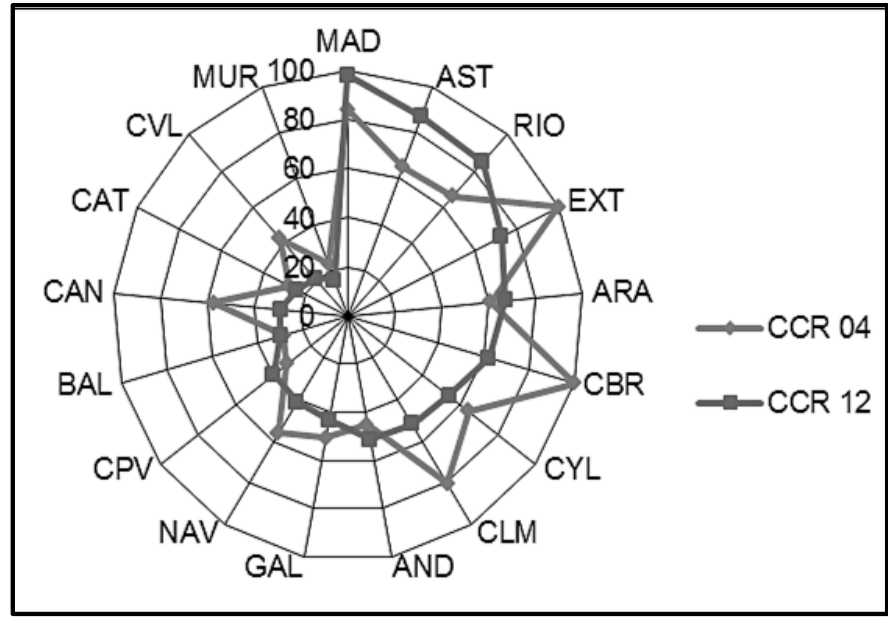

Fuente: Elaboración propia. 
Figura 4

Modelo B. Turismo Cultural Internacional. Evolución de los ratios de eficiencia 2004-2012

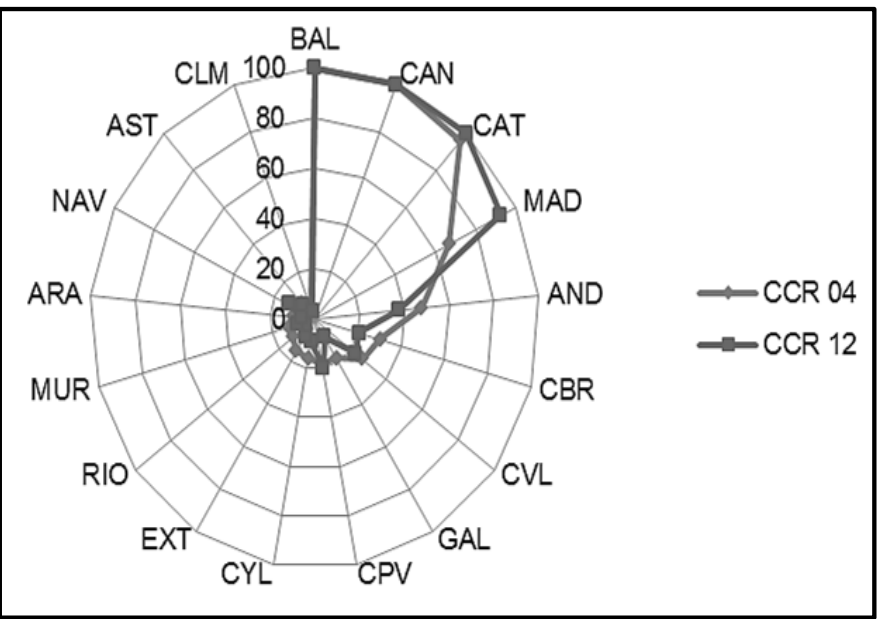

Fuente: Elaboración propia.

Una de las principales aplicaciones del DEA es la posibilidad de obtener información del margen de mejoras posibles de cada DMU, en cada uno de sus inputs y output, a fin de alcanzar la frontera óptima de eficiencia. Podemos ver en la Tabla 3 una medida del porcentaje de mejora estimado. Debido a la orientación hacia la maximización del output, los resultados expresan ajustes necesarios en los factores productivos o aumento pertinente del output, en nuestro caso la entrada de turistas culturales. Mostramos datos medios para todo el periodo analizado, extraídos a partir de las cifras de cada Comunidad Autónoma en cada uno de los 5 años para los que tenemos información. En líneas generales vemos que, para el Modelo A (Tabla 3), las mayores posibilidades de mejora se centran en los museos, donde se manifiesta una cierta sobredimensión en su dotación, para la capacidad de atracción de turismo cultural, que en los casos de Murcia o Castilla la Mancha se estima en un $80 \%$. De igual modo observamos una cierta sobredimensión en la variable empleo cultural, que aún con porcentajes menores, manifiesta unos ajustes en ocasiones superiores al 50\% en determinadas regiones. En cuanto al output, se hace perfectamente patente la necesidad de acrecentar los esfuerzos por parte de todas las Comunidades Autónomas para atraer mayor número de turistas culturales de origen nacional aunque destacan en este sentido las Comunidades del Arco Mediterráneo, como Murcia, donde se debería aumentar el output en más de un $400 \%$ dado el nivel de inputs que presenta, seguida de Cataluña y Comunidad Valenciana, ambas por encima del $200 \%$. 
Tabla 3

Porcentaje de mejora en la gestión de inputs y outputs por regiones (Datos Medios)

\begin{tabular}{|c|c|c|c|c|c|c|c|c|c|c|}
\hline & \multicolumn{5}{|c|}{ MODELO A } & \multicolumn{5}{|c|}{ MODELO B } \\
\hline & Festivales & Museos & BICs & Empleo & \begin{tabular}{|l|} 
Turismo \\
Nacional \\
Cultural
\end{tabular} & Festivales & Museos & BICs & Empleo & $\begin{array}{c}\text { Turismo } \\
\text { Internacional } \\
\text { Cultural }\end{array}$ \\
\hline & $\%$ & $\%$ & $\%$ & $\%$ & $\%$ & $\%$ & $\%$ & $\%$ & $\%$ & $\%$ \\
\hline Andalucía & 0 & $-39,3$ & $-18,5$ & $-40,6$ & 120,7 & $-40,8$ & $-0,1$ & 0 & 0 & 150,8 \\
\hline Aragón & 0 & $-75,4$ & $-39,9$ & $-47,7$ & 53,7 & $-20,9$ & $-32,1$ & 0 & 0 & 1424,6 \\
\hline Asturias & 0 & $-28,3$ & 0 & $-24,2$ & 28,7 & $-42,4$ & $-28,1$ & 0 & 0 & 1322,4 \\
\hline Islas Baleares & 0 & $-72,1$ & $-87,2$ & $-51,1$ & 175,5 & 0 & $-0,0$ & 0 & $-4,2$ & 3,5 \\
\hline Islas Canarias & 0 & $-49,4$ & $-4,7$ & $-62,9$ & 104,4 & 0 & $-2,3$ & 0 & 0 & 4,7 \\
\hline Cantabria & $-5,57$ & $-2,1$ & $-3,9$ & $-10,2$ & 24,2 & $-59,9$ & 0 & 0 & 0 & 301,3 \\
\hline $\begin{array}{c}\text { Castilla y } \\
\text { León }\end{array}$ & 0 & $-76,9$ & $\mid-14,5$ & $-19,9$ & 94,6 & $-47,3$ & $-58,4$ & 0 & 0 & 883,7 \\
\hline $\begin{array}{c}\text { Castilla la } \\
\text { Mancha }\end{array}$ & 0 & $-84,1$ & $\mid-19,6$ & $-35,0$ & 104,0 & $-37,2$ & $-65,5$ & 0 & 0 & 3091,1 \\
\hline Cataluña & $-7,62$ & $-3,0$ & $-0,1$ & $-63,5$ & 276,1 & $-17,3$ & 0 & $-3,6$ & $-24,1$ & 6,9 \\
\hline C. Valenciana & 0 & $-24,2$ & 0 & $-46,4$ & 232,4 & $-27,7$ & $-10,4$ & 0 & 0 & 303,3 \\
\hline Extremadura & 0 & $-31,4$ & 0 & $-2,3$ & 32,9 & $-62,8$ & $-65,1$ & 0 & 0 & 929,9 \\
\hline Galicia & 0 & $-53,2$ & $-2,3$ & $-57,7$ & 94,2 & $-22,3$ & 0 & 0 & 0 & 784,3 \\
\hline Madrid & $-0,14$ & $-15,9$ & 0 & $-9,8$ & 11,9 & $-78,6$ & $-47,4$ & 0 & $-84,3$ & 24,2 \\
\hline Murcia & 0 & $-81,1$ & $-27,8$ & $-51,0$ & 431,2 & $-18,1$ & $-46,3$ & 0 & 0 & 833,1 \\
\hline Navarra & $-14,35$ & 0 & 0 & $-14,9$ & 141,7 & $-46,4$ & $-0,5$ & $-1,7$ & $-3,4$ & 991,6 \\
\hline País Vasco & $-6,85$ & $-5,4$ & 0 & $-2,5$ & 216,9 & $-49,9$ & $-9,8$ & 0 & $-23,3$ & 475,3 \\
\hline La Rioja & $-4,06$ & -4 & 0 & $-22,3$ & 71,6 & $-44,9$ & 0 & 0 & 0 & 1335,7 \\
\hline
\end{tabular}

Fuente: Elaboración propia.

En el Modelo B de nuevo vemos una imagen ciertamente diferenciada respecto al anterior modelo, que ya ocurría en la estimación de los ratios de eficiencia. En primer lugar en cuanto a las mejoras en los inputs, observamos que en este caso el mayor margen de mejora se encuentra en el número de festivales culturales, muy sobredimensionado para el número de turistas internacionales que llegan a estas regiones. Este no parece ser un recurso cultural especialmente atractivo a la hora de atraer turistas extranjeros. En el caso de los museos vemos una situación comparable a lo que mostraban los datos del Modelo A, un número de museos elevado para el nivel de entrada de turistas culturales internacionales. Sin embargo, el número de BICs y el de empleo cultural parecen bastante ajustados al óptimo, en la gran mayoría de regiones. Del lado del output, encontramos que los esfuerzos de mejora deben realizarse especialmente en las regiones del interior, destacando Aragón, La Rioja y Asturias.

Una vez obtenidos los ratios de eficiencia, procedemos en la segunda etapa de evaluación a aplicar el modelo de regresión censurada entre dichos ratios y una serie de variables de entorno, fundamentalmente referidas a condiciones de accesibilidad, recursos complementarios de carácter cultural o natural, capacidad 
hotelera y otros. Es importante considerar que la literatura sobre DEA manifiesta la posibilidad de que las relaciones de eficiencia obtenidas durante la primera etapa pueden estar correlacionadas con las variables explicativas utilizadas durante la segunda etapa, lo que puede conducir a estimaciones sesgadas (Simar y Wilson, 2000). El uso de un procedimiento bootstrap puede ayudar a superar este problema ya que ofrece ciertas mejoras en la estimación. Es por ello que realizamos un proceso de bootstraping con 2000 iteraciones, generando una variable que será la que se incluya como dependiente en el análisis de regresión.

Mostraremos dos resultados para cada tipo de análisis: modelo básico que incluye todas las variables externas consideradas, y modelo depurado que elimina aquellas que carecen de significación estadística según la estimación. Los resultados se muestran en la Tabla 4. Analizamos también los cambios en el signo y el valor de los coeficientes cuando se eliminan ciertas variables del modelo y obtenemos como resultado que éstos apenas varían, lo que nos habla de la homoscedasticidad de los datos.

Tabla 4

Factores externos que influyen en la eficiencia de las regiones. Regresión Censurada

\begin{tabular}{|c|c|c|c|c|c|c|c|c|}
\hline \multirow[b]{3}{*}{ Variables } & \multicolumn{4}{|c|}{ Modelo A (Turismo Cultural Nacional) } & \multicolumn{4}{|c|}{$\begin{array}{l}\text { Modelo B (Turismo Cultural } \\
\text { Internacional) }\end{array}$} \\
\hline & \multicolumn{2}{|c|}{ Modelo Básico } & \multicolumn{2}{|c|}{ Modelo Refinado } & \multicolumn{2}{|c|}{ Modelo Básico } & \multicolumn{2}{|c|}{ Modelo Refinado } \\
\hline & Coeficiente & \begin{tabular}{|l|} 
Error \\
Est.
\end{tabular} & Coeficiente & $\begin{array}{l}\text { Error } \\
\text { Est. }\end{array}$ & Coeficiente & $\begin{array}{l}\text { Error } \\
\text { Est. }\end{array}$ & Coeficiente & $\begin{array}{l}\text { Error } \\
\text { Est. }\end{array}$ \\
\hline Constante & $0.954^{*}$ & 0.118 & $0.834^{*}$ & 0.082 & $-0.833^{\star \star \star}$ & 0.480 & $-1.06^{\star}$ & 0.040 \\
\hline Declaraciones UNESCO & $0.002^{*}$ & 0.000 & $-0.002^{*}$ & 0.000 & -0.000 & 0.002 & - & - \\
\hline Plazas Totales & -0.062 & 0.090 & - & - & $-0.668^{\star}$ & 0.258 & $-0.628^{*}$ & 0.239 \\
\hline Plazas 345 & 0.056 & 0.093 & $-0.009 * \star \star$ & 0.005 & $0.676^{\star \star}$ & 0.268 & $0.631^{\star \star}$ & 0.245 \\
\hline Plazas rural & 0.000 & 0.001 & - & - & 0.001 & 0.003 & - & - \\
\hline Autopistas & $-0.077^{\star}$ & 0.018 & $-0.080^{*}$ & 0.015 & $0.259^{*}$ & 0.075 & $0.270^{*}$ & 0.072 \\
\hline Costa & $-0.015^{\star}$ & 0.003 & $-0.015^{\star}$ & 0.003 & 0.029 ** & 0.012 & $0.030^{*}$ & 0.008 \\
\hline Parques Naturales & $0.011^{*}$ & 0.003 & $0.012^{*}$ & 0.002 & $0.027^{\star \star}$ & 0.011 & $0.027^{\star}$ & 0.006 \\
\hline Gasto Cultural & -0.001 & 0.001 & - & - & -0.003 & 0.004 & - & - \\
\hline Empresas Culturales & $0.004^{*}$ & 0.000 & $0.004^{*}$ & 0.000 & $-0.012^{*}$ & 0.004 & $-0.012^{*}$ & 0.004 \\
\hline Seguridad & $-0.015^{\star \star \star}$ & 0.008 & $-0.011^{\star \star \star}$ & 0.006 & $0.052^{\star \star}$ & 0.026 & $0.056^{\star \star}$ & 0.024 \\
\hline Tendencia & 0.000 & 0.007 & - & - & $-0.062^{\star \star}$ & 0.027 & $-0.059^{\star}$ & 0.023 \\
\hline $\mathrm{N}^{\circ}$ de observaciones & \multicolumn{2}{|l|}{85} & \multicolumn{2}{|l|}{85} & \multicolumn{2}{|l|}{85} & \multicolumn{2}{|l|}{85} \\
\hline $\log L$ & \multicolumn{2}{|l|}{44.26} & \multicolumn{2}{|l|}{43.22} & \multicolumn{2}{|l|}{67.18} & \multicolumn{2}{|c|}{66.78} \\
\hline AIC & \multicolumn{2}{|l|}{-0.73} & \multicolumn{2}{|l|}{-0.80} & \multicolumn{2}{|l|}{-1.27} & \multicolumn{2}{|l|}{-1.33} \\
\hline BIC & \multicolumn{2}{|l|}{-0.36} & \multicolumn{2}{|l|}{-0.54} & \multicolumn{2}{|l|}{-0.90} & \multicolumn{2}{|l|}{-1.04} \\
\hline
\end{tabular}

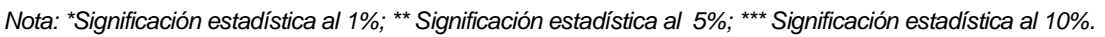

Fuente: Elaboración propia. 
Una vez implementado el modelo, podemos concluir cuáles de las variables exógenas tenidas en cuenta afectan a la eficiencia de los destinos turísticos, así como la medida en que lo condicionan y su sentido, ya sea positivo o negativo. En el denominado Modelo A atendiendo al resultado depurado, observamos que existe una relación inversa de la eficiencia regional en atraer turistas culturales nacionales frente a las variables de accesibilidad, capacidad hotelera y costa. Esto puede interpretarse en el sentido de que el turista cultural nacional se distancia de los destinos congestionados, las zonas identificadas con el turismo denominado de sol y playa y de alta densidad turística, ejemplificado por aquellas regiones que conforman el arco mediterráneo, que son efectivamente poco eficientes en la primera etapa. En este mismo sentido planteamos la interpretación de la variable declaraciones UNESCO, con coeficiente negativo de igual manera, que podría reforzar la idea de que el turista cultural nacional evita, en sus desplazamientos vacacionales, los espacios especialmente notorios y de turismo masivo o bien porque quizás ya conoce los enclaves patrimoniales de más relieve. En cuanto a variables que afectan de forma positiva a la eficiencia, encontramos los parques naturales, que pueden considerarse como un complemento para los turistas nacionales con motivación cultural. Por su parte la variable seguridad también significativa en el modelo refinado, muestra un coeficiente negativo, lo que viene a reafirmar de alguna manera lo anteriormente indicado, el turista nacional evita las zonas típicamente turistas que a la vez son algunas de las que poseen mayor índice de delincuencia. Finalmente la variable empresas culturales obtiene un coeficiente significativo y positivo, lo que indica el alcance que el entramado empresarial cultural, es decir el sector privado, tiene a la hora de incrementar la eficiencia de los destinos turísticos.

En el Modelo B (turismo cultural internacional) observamos un escenario donde los signos de los coeficientes de un buen número de variables son inversos a los anteriormente interpretados. Si de nuevo atendemos al modelo final refinado advertimos que, frente a lo que ocurría en el Modelo A, la variable autopistas en este caso presenta un signo positivo, es decir que los turistas internacionales buscan regiones con mejores y más fáciles accesos. Además observamos un carácter omnívoro en la motivación de la elección de destino por parte de estos turistas, ya que les condiciona de forma positiva la presencia de litoral y de parques naturales. Esto constata que, aunque practican una intencionalidad de participación cultural en su visita turística, realizan otro tipo actividades de ocio alternativas. Interesante es la significatividad de la variable seguridad, en el caso de los turistas internacionales, no solo afecta, sino que lo hace de forma positiva. En un primer momento podía parecer contradictorio, sin embargo hemos interpretado este signo como una relación entre la existencia de delitos y las zonas de mayor concentración turística. En cuanto a coeficientes negativos a la hora de atraer turistas internacionales, nos encontramos con la variable tendencia, que nos habla del paso del tiempo en el 
modelo analizado. Podemos entender que este coeficiente significativo y negativo, es la manifestación de un proceso de maduración de los destinos turísticos acreditados, a medida que pasa el tiempo estos se vuelven menos eficientes. Por otro lado analizamos de forma conjunta las variables plazas totales y plazas 3, 4 y 5 estrellas. En el caso de la primera su signo es negativo, mientras que la segunda es positiva. Esto indica que los turistas de origen internacional acuden a aquellas regiones donde la disponibilidad de plazas hoteleras en establecimiento de mayor categoría es más abundante, mientras que si en la variable incluimos los hoteles de 2 y 1 estrellas, el coeficiente pasa a ser negativo. En último lugar, la presencia de empresas culturales influye de forma negativa para la asistencia de turistas internacional, esto nos hace pensar en que es un turismo más ligado a turoperadores, donde se realiza la contratación de una oferta completa, no haciendo especial uso de la infraestructura privada de servicios culturales en las regiones que visitan.

\section{CONCLUSIONES}

Hemos constatado que es posible realizar un estudio que mide la eficiencia de los destinos regionales de turismo cultural, así como los determinantes de la misma, por medio de un análisis de eficiencia en dos etapas (DEA y modelo de regresión censurada). Se han aplicado sobre el contexto de las regiones españolas dos modelos, cuya principal diferencia radica en el output de la función de producción diseñada; el modelo $\mathrm{A}$, analiza la eficiencia de los destinos en la captación de turismo cultural nacional y el modelo B aborda el supuesto del turismo cultural internacional. Se consideran a modo de factores productivos las diferentes dotaciones en capital cultural, y el factor trabajo propio del sector cultural. De la aplicación del análisis DEA se han obtenido los ratios de eficiencia para cada modelo.

Tras observar qué regiones son eficientes, podemos concluir que existe una tendencia inversa en la eficiencia de los destinos turísticos, si se tiene en cuenta el origen de los turistas. Mientras que las regiones más eficientes para el turismo cultural nacional son las de interior y ciertos casos de la costa cantábrica, en el caso del turismo internacional destacan por su nivel de eficiencia regiones del litoral, especialmente del Mediterráneo y las insulares, aquellas con una acreditación turística más elevada. Posteriormente estos ratios han sido introducidos como variable dependiente en un análisis de regresión censurada, a fin de contrastar qué variables externas, es decir no controlables, afectan a su eficiencia. Consideramos como variables exógenas las representativas de la accesibilidad, dotaciones hoteleras, alternativas de ocio, y la existencia de litoral y parques naturales y la presencia de un mayor entramado de empresas dedicadas al sector. De todo ello hemos podido extraer que pese a desplegar los mismos recursos, los destinos turísticos muestran un nivel de eficiencia diferente, 
dependiendo del origen de los turistas. En líneas generales los destinos que atraen con mayor eficiencia a los turistas internacionales, ponen de manifiesto un mayor margen de mejora en la atracción de turistas nacionales y viceversa. Esto nos habla de dos segmentos de turismo distintos, el turista internacional muestra un patrón omnívoro, pues aunque realice consumos culturales y así lo declara, se ve atraído por la presencia de costa y parques naturales y se dirige a las regiones de mayor congestión turística en general. Sin embargo los turistas culturales nacionales presentan como determinantes los recursos naturales y muestran un claro sentido opuesto a la presencia de masificación turística, para este flujo se muestran más eficientes las regiones del interior y algunas del Cantábrico. Los resultados parecen estar reflejando la diversificación de viajes que realizan los turistas culturales nacionales. Estos plantean diferentes viajes uni-propósito según su motivación, cultural o no cultural, no combinando ambos motivos en una única salida. Por su parte, los turistas internacionales, trataran de combinar ambas tendencias en un único viaje, algo que resulta obvio teniendo en cuenta el mayor esfuerzo económico requerido y la multiplicidad de destinos alternativos.

Consideramos que las conclusiones extraídas del presente estudio pueden tener una aplicabilidad relevante dentro del sector de las políticas turísticas y culturales. De la discusión aquí generada pueden derivarse toda una serie de herramientas con utilidad en el proceso de toma de decisiones, dentro de la rama de la gestión cultural y turística. La principal utilidad vendrá dada en tanto que se han focalizado los factores y recursos regionales a los que se debe atender en mayor medida, si lo que se pretende es incrementar el volumen de entrada de turistas culturales. Por otro lado debe señalarse también que de este tipo de análisis podemos extraer un mejor conocimiento sobre la distribución a nivel regional de la oferta y demanda turística con naturaleza cultural en nuestro país. Finalmente nos permite comprobar el desempeño del esfuerzo realizado, a nivel público, en la creación de dotaciones culturales y otros recursos turísticos relacionados, en términos de resultados sobre entradas de flujos turísticos de rango nacional o internacional.

\section{REFERENCIAS BIBLIOGRÁFICAS}

ÁLVAREZ, A. (2001). La medición de la eficiencia y la productividad. Madrid: Pirámide. ASSAF, A.G. y AGBOLA, F.W. (2011). "Modelling the performance of Australian Hotels: a DEA doublé bootstrap approach". Tourism economics, 17(1), pp. 73-89.

ASSAF, A.G. y CVELBAR, L.K. (2010). "The performance of Slovenian hotel industry: evaluation post-privatisation". International Journal of Tourism Research, 7(3), pp. 173-184. 
BANKER, R.D. y NATARAJAN, R. (2008). "Evaluating Contextual Variables Affecting Productivity using Data Envelopment Analysis". Operations Research, 56(1), pp. 4858.

BARBIERI, C. y MAHONEY, E. (2010). "Cultural tourism behaviour and preferences among the live performing arts audience: an application of the univorous-omnivorous framework". International Journal of Tourism Research, 12(5), pp. 481-496.

BARROS, C.P. (2004). "A stochastic cost frontier in the Portuguese hotel industry". Tourism Economics, 10(2), pp. 177-192.

BARROS, C.P. (2005). "Measuring efficiency in the hotel sector". Annals of Tourism Research, 322, pp. 456-477.

BARROS, C.P. (2006). "Analyzing the rate of technical change in the Portuguese hotel industry". Tourism Economics, 12(3), pp. 325-346.

BARROS, C.P. y MATIAS, Á. (2006). "Assessing the efficiency of travel agencies with a stochastic cost frontier: a Portuguese case study". International Journal of Tourism Research, 8(5), pp. 367-379.

BARROS, C.P.; BOTTI, L.; PEYPOCH, N.; ROBINOT, E.; SOLONANDRASANA, B. y ASSAF, A. (2011). "Performance of French destinations: Tourism Attraction Perspectives". Tourism Management, 32, pp.141-146.

BASSO, A. y FUNARI, S. (2004). "A Quantitative Approach to Evaluate the Relative Efficiency of Museums". Journal of Cultural Economics, 28(3), pp. 195-216.

BENITO, B.; SOLANA, J. y LÓPEZ, P. (2014). "Determinants of Spanish regions' tourism performance: a two-stage, double-bootstrap, data envelopment analysis". Tourism economics, 20(5), pp. 987-1012.

BISHOP, P. y BRAND, S. (2003). "The Efficiency of Museums: A Stochastic Frontier Production Function Approach". Applied Economics, 35(17), pp. 1853-1858.

BOTTI, L.; PEYPOCH, N.; ROBINOT, E. y SOLONANDRASANA, B. (2009). "Tourism destination competitiveness: the French regions case". European Journal of Tourism Research, 21, pp. 5-24.

BRIDA, J.G., DEIDDA, M., GARRIDO, N. y MANUELA, P. (2015). "Analyzing the performance of the South Tyrolean hospitality sector: a dynamic approach". International Journal of Tourism Research, 17(2), pp. 196-202.

CASTRODEZA, C. y PEÑA, T. (2002). "Evaluación de la actividad investigadora universitaria: Una aplicación a la Universidad de Valladolid". Estudios de Economía Aplicada, 20(1), pp. 29-44.

CRACOLICI, M.F.; NIJKAMP, P. y RIETVELD, P. (2008). "Assessment of tourism competitiveness by analysing destination efficiency". Tourism Economics, 14, pp. 325-342.

CUCCIA, T. y CELLINI, R. (2006). "Is cultural heritage really important for tourists? A contingent rating study". Applied Economics, 39(2), pp. 261-271.

CUCCIA, T.; GUCCIO, C. y RIZZO, I. (2013). "The effects of UNESCO world heritage list inscription on tourism destinations performance in italian regions". Economic Modeling, 53, pp. 494-490.

DARAIO, C. y SIMAR, L. (2005). "Introducing Environmental Variables in Nonparametric Frontier Models: A Probabilistic Approach". Journal of Productivity Analysis, 24(1), pp. 93-121. 
DE WITTE, K. y GEYS, B. (2011). "Evaluating Efficient Public Good Provision: Theory and Evidence from a Generalised Conditional Efficiency Model for Public Libraries". Journal of Urban Economics, 69(3), pp. 319-327.

DEL BARRIO, M.J. y HERRERO, L.C. (2014). "Evaluating the efficiency of museums using multiple outputs: evidence from a regional system of museums in Spain". International Journal of Cultural Policy, 20(2), pp. 221-238.

DEL BARRIO, M.J.; HERRERO, L.C. y SANZ, J.A. (2009). "Measuring the Efficiency of Heritage Institutions: A Case Study of a Regional System of Museums in Spain". Journal of Cultural Heritage, 10(2), pp. 258-268.

DIOS, R.; MARTÍNEZ, J.M. y MARTÍNEZ-CARRASCO, F. (2006). "El análisis de eficiencia con variables de entorno: un método de programas con tres etapas". Estudios de Economía Aplicada, 24(1), pp. 477-497.

FERNÁNDEZ BLANCO, V., HERRERO PRIETO, L.C. y PRIETO RODRÍGUEZ J. (2013), "Performance of Cultural Heritage Institutions". En RIZZO, I. y MIGNOSA, A., (eds): Handbook on Economics of Cultural Heritage. Edward Elgar Publishing Ltd, Cheltenham, UK and Northampton, MA, USA.

FINOCCHIARO, M., GUCCIO, C. y RIZZO, I. (2011). "Public intervention on heritage conservation and determinants of heritage authorities' performance: a semiparametric analysis". International tax and public finance, 18819, pp. 1-16.

FUENTES, R. (2011). "Efficiency of travel agencies: A case study of Alicante, Spain". Tourism Management, 32, pp. 75-87.

HERRERO, L.C. y GÓMEZ, M. (2017). "Cultural resources as a factor in cultural tourism attraction. Technical efficiency estimation of regional destinations in Spain". Tourism Economics, 23(2), pp. 260-280.

KÖKSAL, C.D. y AKSU, A.A. (2007). "Efficiency evaluation of A-group travel agencies with data envelopment analysis (DEA): a case study in the Antalya region, Turkey". Tourism Management, 28, pp. 830-834.

LAST, A. K. y WETZEL, H. (2010). "The Efficiency of German Public Theaters: A Stochastic Frontier Analysis Approach". Journal of Cultural Economics, 34(2), pp. 89110.

MAIRESSE, F. y VANDEN EECKAUT, P. (2002). "Museum Assessment and Fdh Technology: Towards a Global Approach". Journal of Cultural Economics, 26(4), pp. 261-286.

MARTÍNEZ, M. (2003). La medición de la eficiencia en las instituciones de educación superior. Madrid: Fundación BBVA.

MINISTERIO DE EDUCACIÓN, CULTURA Y DEPORTE. (2013). Anuario de Estadísticas Culturales. Madrid: Secretaría General Técnica. Ministerio de Educación, Cultura y Deporte.

PIGNATARO, G. (2002). "Measuring the Efficiency of Museums: A Case Study in Sicily". En Rizzo I. y Towse R. (eds.): The Economics of Heritage. A Study in the Political Economy of Culture in Sicily (pp. 65-78). Cheltenham, UK and Northampton, MA, USA: Edward Elgar Publishing Ltd.

QUINDÓS, M.P.; CUEVA, M.R. y RUBIERA, F. (2005). "La eficiencia de las actividades de I+D desde el punto de vista de las patentes registradas en los países de la Unión Europea". Estudios de Economía Aplicada, 23(3), pp. 607-619.

RIERA, A.; RIPOLL, A.M. y JUANEDA, C.N. (2011). "Efficiency and Seasonality in the Balearic Hospitality Industry". Estudios de Economía Aplicada, 23(3), pp. 845-862. 
SAINAGHI, R.; PHILLIPS, P. y ZAVARRONE, E. (2017). "Performance measurement in tourism firms: A content analytical meta-approach". Tourism Management, 59, pp. 36-56.

SHANG, J.K.; WANG, F.C. y HUNG, W.T. (2010). "A stochastic DEA study of hotel efficiency". Applied Economics, 42, pp. 2505-2518.

SIGALA, M. (2004). "Using data envelopment analysis for measuring and benchmarking productivity in the hotel sector". Journal of Travel \& Tourism Marketing, 16(2/3), pp. 3960.

SIMAR, L. y WILSON, P. (2000). "A general methodology for bootstrapping in nonparametric frontier models". Journal of Applied Statistics, 27, pp. 779-802.

SIMAR, L., y WILSON, P.W. (2007). "Estimation and inference in two-stage, semiparametric models of production processes". Journal of Econometrics, 136, pp. 31-64.

SUZUKY, S.; NIJKAMP, P. y RIETVELD, P. (2011). "Regional efficiency improvement by means of data envelopment analysis through Euclidean distance minimization including fixed input factors: An application to tourist regions in Italy". Papers in Regional Science, 90(1), pp. 67-89.

URUEÑA, B. y NIÑO, N. (2012). "La evaluación de la eficiencia en las universidades: Un análisis de inputs y outputs por áreas de conocimiento". Estudios Económicos Regionales y Sectoriales, 12(3), pp. 209-225.

VITALIANO, D.F. (1998). "Assessing Public Library Efficiency Using Data Envelopment Analysis". Annals of Public and Cooperative Economics, 69(1), pp. 107-122.

WANG, F.C.; HUNG, W.T. y SHANG, J.K. (2006). "Measuring the cost efficiency of international tourist hotels in Taiwan". Tourism Economics, 121, pp. 65-85.

ZIEBA, M. (2013). "Evaluación de la eficiencia de las artes escénicas en Alemania, Austria y Suiza mediante métodos paramétricos". En Herrero L.C. (ed.): Evaluación de la eficiencia de instituciones culturales (pp. 81- 114). Valladolid: Fundación del Patrimonio Histórico de Castilla y León. 